\title{
BMJ Open Australian children undergoing selective dorsal rhizotomy: protocol for a national registry of multidimensional outcomes
}

\author{
Jennifer Lewis, ${ }^{1}$ Natasha Bear, ${ }^{2}$ Felicity Baker, ${ }^{3}$ Adam Fowler, ${ }^{4}$ Olivia Lee, ${ }^{5}$ \\ Kim McLennan, ${ }^{6}$ Emma Richardson, ${ }^{7}$ Adam Scheinberg, ${ }^{5,8}$ Nadine Smith, ${ }^{7}$ \\ Pam Thomason, ${ }^{9}$ Andrew Tidemann, ${ }^{10}$ Meredith Wynter, ${ }^{6}$ Simon Paget ${ }^{11,12}$
}

To cite: Lewis J, Bear N, Baker $\mathrm{F}$, et al. Australian children undergoing selective dorsal rhizotomy: protocol for a national registry of multidimensional outcomes. BMJ Open 2019;9:e025093. doi:10.1136/ bmjopen-2018-025093

- Prepublication history for this paper is available online. To view these files, please visit the journal online (http://dx.doi org/10.1136/bmjopen-2018025093).

Received 4 July 2018 Revised 12 February 2019 Accepted 13 February 2019

Check for updates

(c) Author(s) (or their employer(s)) 2019. Re-use permitted under CC BY-NC. No commercial re-use. See rights and permissions. Published by BMJ.

For numbered affiliations see end of article.

\section{Correspondence to}

Ms Jennifer Lewis;

jennifer.lewis@health.nsw. gov.au

\section{ABSTRACT}

Introduction Selective dorsal rhizotomy (SDR) is a neurosurgical intervention intended to permanently reduce spasticity in the lower limbs and improve mobility in selected children with cerebral palsy (CP). Despite SDR having been performed worldwide for the past 30 years, there is moderate quality of evidence that SDR is effective in reducing spasticity with low to very low evidence of its effectiveness in improving gait, function and participation, using the Grading of Recommendations, Assessment, Development and Evaluations (GRADE) system. Published studies have described outcomes for groups that differ in selection, surgical technique and postoperative rehabilitation making it difficult for clinicians to use this information to advise families on best management. There is substantial community interest in SDR. A small number of children with CP undergo SDR in Australia each year and some families seek the intervention at international sites. Capturing clinical outcomes and adverse event (AE) data for Australian children undergoing SDR will provide clinicians with information to help guide families considering SDR.

Methods and analysis The Australian SDR Research Registry is a national registry of multidimensional outcomes for Australian children undergoing SDR in an Australian or overseas centre. Data will be collected for up to 10 years following the surgery, to include surgery and admission details, surgical and long-term AEs, and outcome measures across the body structure and functions, activity and participation domains of the International Classification of Functioning, Disability and Health. Data will be collected at baseline, during inpatient admission and at 1, 2, 5 and 10 years post. The aim of collecting these data is to improve understanding of short-, medium- and long-term outcomes and adverse effects of the intervention.

Ethics and dissemination This study was approved by the individual Human Research and Ethics committees at the five Australian tertiary hospitals involved. Results will be disseminated via peer-reviewed publications and conference presentations.

Trial registration number ACTRN12618000985280; Preresults.

\section{INTRODUCTION}

Cerebral palsy (CP) is an umbrella term to describe a group of permanent disorders
Strengths and limitations of this study

- A population-based study investigating short-, medium- and long-term outcomes of children undergoing selective dorsal rhizotomy (SDR) in Australia and overseas.

- Data collection of outcome measures across all the domains of the International Classification of Functioning, Disability and Health.

- Data collection of perioperative and long-term adverse events to allow monitoring of ongoing safety and efficacy.

- Study protocol includes retrospective and prospective data. Limitation as a result of missing data, particularly for children going overseas for SDR surgery, may occur.

- The Australian population base and this study design does not enable the inclusion of a control group for comparison.

affecting the development of movement and posture and causing activity limitations, which is due to nonprogressive damage to the developing brain in utero, during birth or in the first years of life. ${ }^{1} \mathrm{CP}$ is the most common cause of physical disability in children worldwide. The incidence of CP in Australia and Europe has remained stable in recent years at $1.5-2.5 / 1000$ live births. ${ }^{23}$

Spasticity is the predominant motor type in CP $(85.8 \%){ }^{4}$ "Spasticity is defined as hypertonia in which one or both of the following signs are present: (1) resistance to externally imposed movement increases with increasing speed of stretch and varies with the direction of joint movement and/or (2) resistance to externally imposed movement rises rapidly above a threshold speed or joint angle". ${ }^{5}$ Management of CP often involves interventions that reduce spasticity including oral medications, injectable botulinum toxin A, neurosurgery and orthopaedic surgery. ${ }^{6}$ Interventions should 
be chosen with consideration of 'negative features' of CP including muscle weakness, loss of dexterity and selective motor control, fatigue and dyspraxia which often occur in conjunction with spasticity. ${ }^{7}$ Treatment goals are often also dependent on other factors including comorbidities, age of the child, cooperation of the child, access to rehabilitation services and expectations of the parents/carers.

Selective dorsal rhizotomy (SDR) is a neurosurgical intervention performed to reduce spasticity in the lower limbs. ${ }^{8}$ This is achieved through the transection of a proportion of the dorsal (sensory) nerve rootlets in the lumbar spine, thus interrupting the afferent input of the monosynaptic stretch reflex. SDR results in a permanent reduction in spasticity and the effects are irreversible. SDR has been most commonly used as an adjunctive procedure (ie, supplementing other therapies and interventions) for children with spastic diplegia who walk unaided or using a walking device.

SDR has a long history dating back to $1908 .^{9}$ The surgery was associated with frequent surgical complications and became a less favourable intervention option until Fasano introduced partial sectioning of the dorsal nerve rootlets on the basis of intraoperative electrophysiological stimulation in 1978. This resulted in better outcomes and increased interest in the use of the procedure for children with CP in the 1980s. Studies from this time suggested SDR to be of most benefit for young children with spastic diplegic CP, without dyskinesia or ataxia, without significant cognitive disability and with emerging/established walking capacity or ambulation (table 1).$^{10}$ There is moderate evidence that SDR is effective in reducing spasticity, with low to very low evidence of its effectiveness in improving gait, function and participation. ${ }^{11}$ In view of this, SDR remains controversial as much remains unknown about the procedure, particularly with respect to selection criteria and long-term outcomes.

\section{Evidence for SDR in children}

Selection criteria for SDR vary between centres and have not been generally validated, as illustrated in a recent systematic review. ${ }^{12}$ The general selection criteria defined by Peacock in $1987^{10}$ still apply but more recent studies have examined SDR being performed in children with severe spasticity in Gross Motor Functional Classification System (GMFCS) levels IV and V, as a cost-effective intervention for the goals of improved care and comfort. ${ }^{13-15}$

Studies conducted in assessing the effectiveness of SDR in children with CP have been mostly of evidence levels III and IV. ${ }^{16}$ Published studies have described outcomes for groups that differ in selection, surgical technique and postoperative rehabilitation. Follow-up periods range from 6 months ${ }^{17}$ up to 26 years. ${ }^{18} 19$ Outcome measures in published studies have primarily drawn from the International Classification of Functioning, Disability and Health (ICF) domains of body structure and functions ${ }^{2021}$ (modified Ashworth Scale, range of motion, gait parameters, pain) and activities ${ }^{22-31}$ (Gross Motor Function Measure, GMFM). The participation domain, quality of life and personal factors have seldom been reported on other than in nonstandardised surveys and/or interviews, ${ }^{26} 3233$ with the exception of Langerak who used the Life-Habit questionnaire to measure activity and participation. ${ }^{18}$

There is also a need to better understand potential risks and complications related to SDR. Adverse events (AEs) have been reported in the literature as short- and longterm complications. Short-term complications reported postoperatively have included spasms, hypotonia, urinary incontinence, headache, wound healing and hyperaesthesia. ${ }^{3134}$ A systematic review of studies documenting long-term AEs post-SDR suggested the presence of spinal abnormalities and/or back pain to be the most commonly reported AEs. ${ }^{35}$ It is unclear to what extent SDR contributes to the spinal abnormalities described as no comparison was made to matched controls in any of the studies. ${ }^{36-39}$

\section{SDR in Australia}

Current SDR surgery programmes began in Australia in 2003. Neurosurgeons at the Children's Hospital at Westmead (CHW) Sydney, the Royal Children's Hospital (RCH) Melbourne and Lady Cilento Children's Hospital (LCCH; formerly $\mathrm{RCH}$ ) Brisbane were trained by a visiting neurosurgeon affiliated with Gillette Children's Specialty Healthcare and The Shriners Hospitals for Children, Minnesota. Protocols pertaining to surgical procedure (intraoperative monitoring and anaesthetic protocol) and postoperative rehabilitation were adopted from those developed at these facilities. Selection criteria were informed by those provided by Peacock $e t$ al as benefiting most by the intervention (table 1). ${ }^{10}$ Currently, most children selected for SDR are between 4 and 8 years.

In 2012, a multidisciplinary working party was formed to address the need for standardised evaluations at key times for children undergoing SDR in Australia, to ensure that data pooling in the future would be possible. This dataset resulted in an agreed schedule of assessments (minimum dataset) for long-term follow-up post-SDR. This was further refined with the establishment of the Australian SDR (SDR-AUS) Research Registry committee in 2014

Table 1 Preference for patient selection criteria for SDR

\begin{tabular}{ll}
\hline Indications for SDR & Contraindications for SDR \\
\hline Spastic CP, affecting predominantly the lower limbs & Presence of severe contractures \\
GMFCS level II or III & Increased weakness in antigravity muscles \\
Capacity to engage in intense rehabilitation programme & Marked presence of other movement disorders (ataxia, \\
No previous orthopaedic surgeries & dystonia, choreoathetosis) \\
\hline
\end{tabular}

CP, cerebral palsy; GMFCS, Gross Motor Function Classification Scale; SDR, selective dorsal rhizotomy. 
consisting of medical, surgical and allied health (physiotherapists) clinicians from all of the tertiary paediatric hospitals involved in SDR management in Australia. The main purpose of the committee was to establish an agreed national SDR assessment protocol and AEs registry. This registry is to include details of the surgery and surgical complications as well as long-term AEs and outcome measures related to functional mobility and activity, goal attainment and quality of life. The protocol for outcome measures is based on the best available evidence of assessment tools that capture all domains of the ICF. As the numbers of children undergoing SDR in Australia is small, the benefits in having a multicentre study to pool data were recognised. A set of research questions were identified by the Registry committee.

Data collected in the registry will be used to answer the following research questions:

1. What are the characteristics of Australian children with $\mathrm{CP}$ who have undergone SDR?

2. How do gait, gross motor function and mobility change 12 months, 2 years, 5 years and 10 years following SDR.

a. How does GMFCS level affect changes in gait, gross motor function and mobility change 12 months, 2 years, 5 years and 10 years following SDR.

b. How do changes in gait, gross motor function and mobility 12 months, 2 years, 5 years and 10 years following SDR differ between children who have had the procedure performed in Australia compared with overseas.

3. What are the functional goal-directed outcomes of children who undergo SDR (in Australia) and how effective is SDR for goal attainment at 1 and 2 years after procedure.

a. How does GMFCS level at time of surgery impact on goal setting before surgery and on goal attainment at 1 and 2 years post-SDR.

b. How do changes in goal attainment compare with changes in gross mobility.

4. What (types and frequency of) spasticity management and orthopaedic interventions are required in 10 years following SDR?

a. How does GMFCS level affect (types and frequency of) spasticity management and orthopaedic interventions required in 10 years following SDR?

b. How do children who have undergone SDR in Australia compare with those who have had the procedure overseas with respect to (types and frequency of) spasticity management and orthopaedic interventions required in 10 years following SDR?

5. How common and what type and severity of surgical complications occur following SDR surgery?

a. How does GMFCS level affect the frequency, type and severity of surgical complications occur following SDR surgery?

6. What is the prevalence of long-term AE at 12 months, 2 years, 5 years and 10 years following SDR? a. How does GMFCS level affect the prevalence of long-term $\mathrm{AE}$ at 12 months, 2 years, 5 years and 10 years following SDR.

b. How does site of surgery (Australia vs overseas) affect the prevalence of (the above long-term AE) at 12 months, 2 years, 5 years and 10 years following SDR?

7. What is the experience of pain of children at baseline, 1 year, 2 years, 5 years and 10 years following SDR?

a. How does GMFCS affect the experience of pain of children at baseline, 1 year, 2 years, 5 years and 10 years following SDR?

b. How does site of surgery (Australia vs overseas) affect experience of pain of children at baseline, 1 year, 2 years, 5 years and 10 years following SDR?

8. What is the quality of life for children (proxy report) at 2 years, 5 years and 10 years following SDR?

a. How does GMFCS affect the quality of life for children (proxy report) at 2 years, 5 years and 10 years following SDR?

b. How does site of surgery (Australia vs overseas) affect the quality of life for children (self or proxy report) at 2 years, 5 years and 10 years following SDR?

\section{METHODS AND ANALYSIS}

This study is a national registry of multidimensional outcomes for Australian children undergoing SDR surgery. Children will be recruited across multiple tertiary paediatric centres in Australia. Data collected will be from clinical assessments performed by experienced clinicians at these centres prior to and following SDR and will include diagnostic and classification data, clinical assessment, results of several standardised tools and the results of radiological investigations ordered as part of children's routine care. Data collected will be entered by study investigators into a purpose-designed research database.

\section{Study population and recruitment}

Study participants will be recruited from (a) children who have previously undergone SDR in an Australian or overseas centre and (b) children who are scheduled to undergo SDR in an Australian or overseas centre. Families will be approached about the study at the time of a clinic review prior to or in follow-up from SDR. A brief verbal introduction to the study will be given (by a clinician and/or study investigator) and followed up with an information sheet and consent form. Families will be asked to consider participation and, if willing, to pass the signed consent form to the research team, either at the time of discussion or at any point during the hospital admission for SDR. Families who do not wish to participate will be asked to inform the researcher so as not to be asked again. Families will be assured that nonparticipation will not make any difference to their child's clinical care. The described dataset are all current standard of care for children undergoing SDR in Australia. 


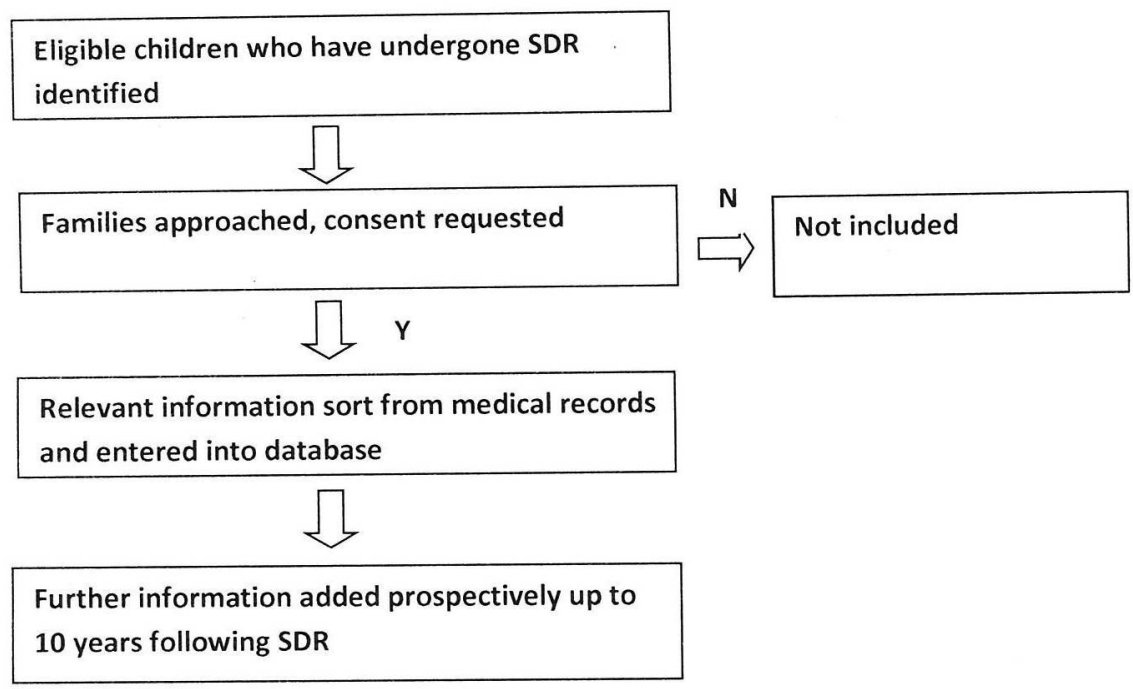

Figure 1 Retrospective recruitment of children who have undergone SDR prior to the commencement of study. SDR, selective dorsal rhizotomy.

The hospitals currently participating in the SDR-AUS registry study include The CHW, Sydney, New South Wales; The Princess Margaret Hospital for Children, Perth, Western Australia; The RCH, Melbourne, Victoria; LCCH, Brisbane, Queensland and The Women's and Children's Hospital Adelaide, South Australia.

Recruitment will be retrospective and prospective as detailed in figures 1 and 2.

\section{Outcome measures}

Data will be collected during three periods of time (table 2):

- At the time of selection and baseline

- At the time of SDR and postoperatively

- During rehabilitation and long-term follow-up to 10 years for complete dataset collection prior to transitioning to adult services.

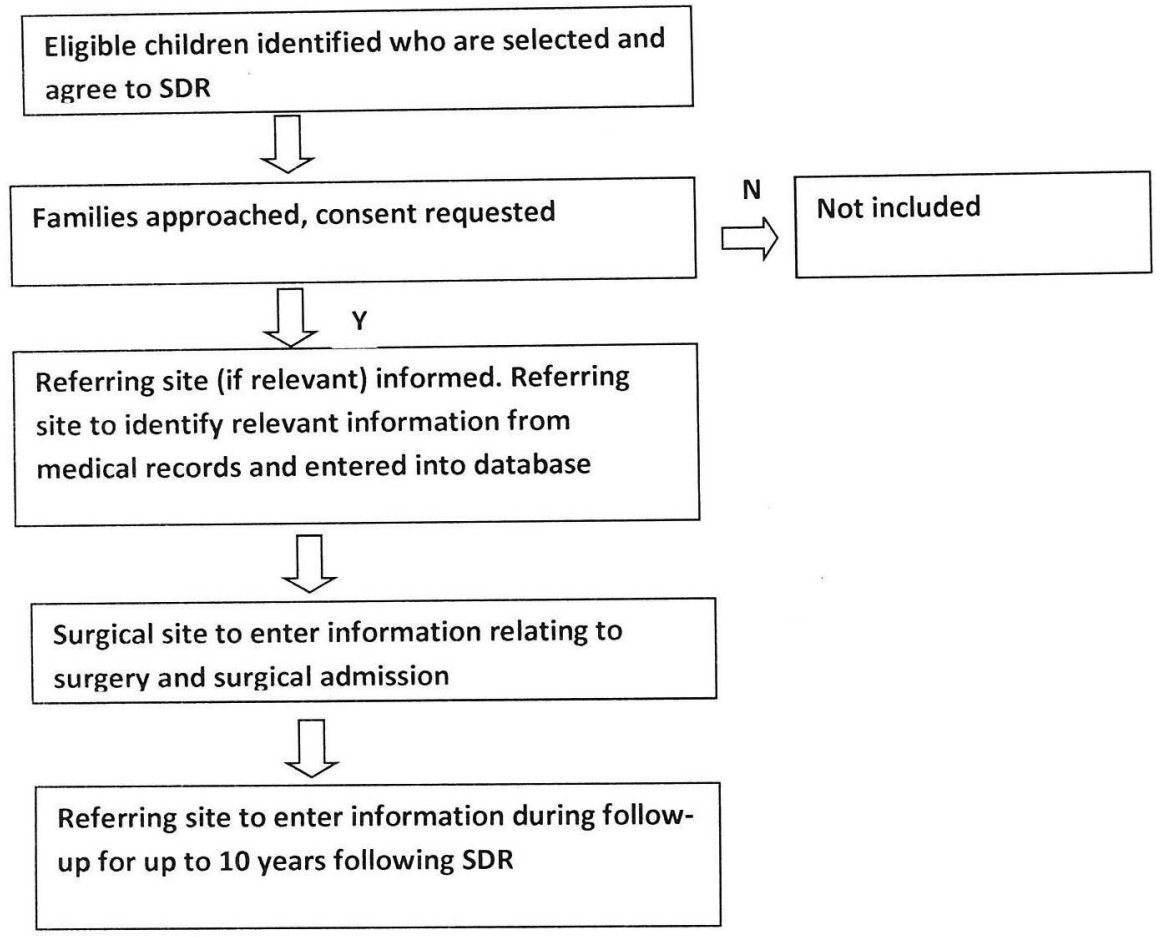

Figure 2 Prospective recruitment of children undergoing SDR following commencement of study. SDR, selective dorsal rhizotomy. 
Table 2 Timing of data collection for SDR-AUS registry study

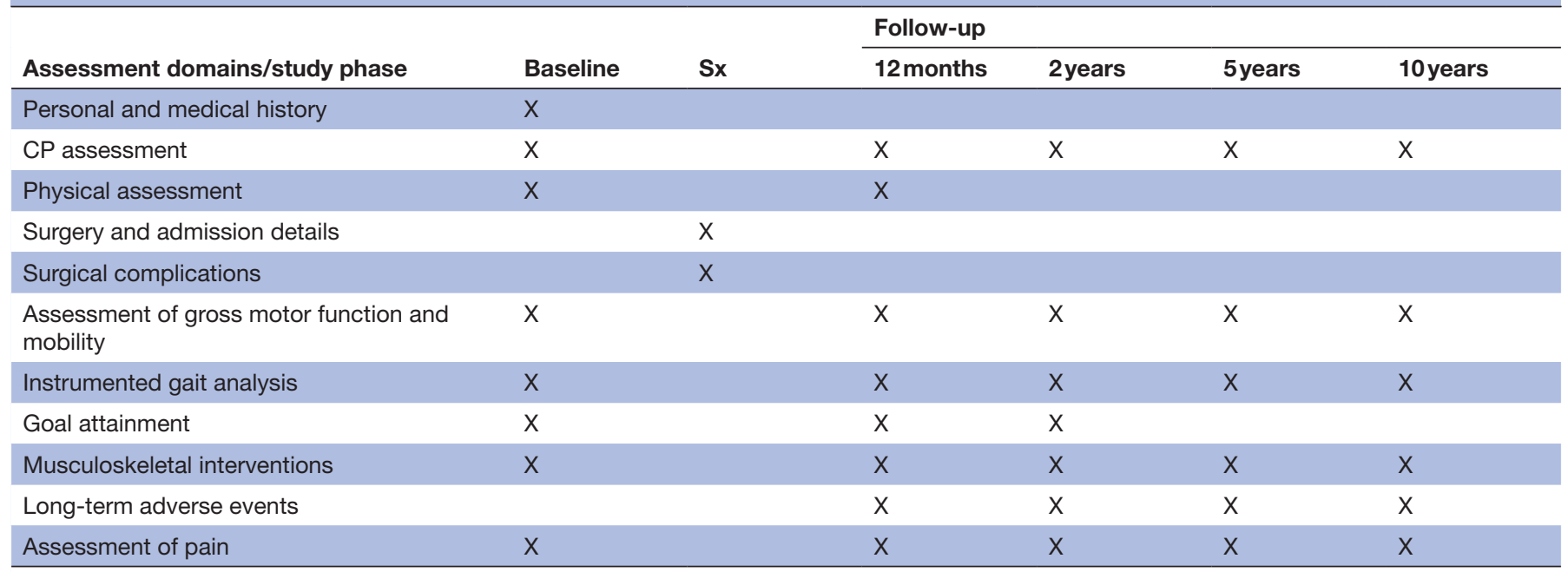

CP, cerebral palsy; SDR-AUS, Australian selective dorsal rhizotomy; Sx, surgical.

The selection of outcome measures and standardised assessment tools have been chosen to reflect the domains of the ICF and developed for the CP and/or paediatric population (table 3). The Data Collection Assessment Manual outlining the dataset is uploaded onto the REDCap database and describes each assessment, when and how it is administered and recorded in the database. This will enable consistency in collection of outcomes, allow for comparison of studies in the literature and further enhance literature of SDR in the paediatric population.

\section{Participant background and demographic data}

Data to be collected include

Personal and medical details: gender, date of birth, state/territory of residence, postcode, diagnosis, gestation at birth and results of neuroimaging (MRI of the brain and spine, as available).
CP assessment: predominant movement disorder (spasticity/dystonia/mixed/other), indication of the presence of dystonia using the Hypertonia Assessment Tool, topography (hemiplegia/diplegia/ quadriplegia) and classification of the sample according to gross motor function (GMFCS), function of the upper extremities (Manual Abilities Classification System) and communication with familiar and unfamiliar partners (Communication Function Classification System).

\section{Details of the intervention and immediate postoperative period}

Data to be collected include

Surgery and admission: date of surgery, site of surgery (CHW/RCH/Overseas/Other), surgical approach (multilevel laminectomy/multilevel laminoplasty/conus

Table 3 The primary and secondary outcome measures

\section{Gross motor function and mobility} (activity)
Gross motor function measure-66

Functional Mobility Scale

Gillette Functional Assessment Questionnaire

Six min walk test

Timed up-and-go
Gait (body structure and functions)

Goal attainment (activity and participation)

Musculoskeletal interventions (body structure and functions)

Pain (body structure and functions)

Gait Profile Score and Gait Variable Scores from 3DGA (barefoot)

\section{Canadian Occupational Performance Measure}

Botulinum toxin A injections

Musculoskeletal surgeries

Faces Pain Scale-Revised

Checklist of pain location

PROMIS Paediatric Pain Interference Scale

Quality of life (personal factors) 
approach laminotomy), presence of electrophysiological guidance, total rootlets transected (laterality).

Surgical complications: type of surgical/postoperative complication during the inpatient period and classification according to Clavien-Dindo classification. ${ }^{40}$

\section{Long-term AEs}

Data to be collected include the following

Presence of skeletal deformity: This includes spinal deformity (presence and degree of lordosis, scoliosis, kyphosis, spondylolisthesis and spondylosis), hip subluxation (Reimers' migration percentage) and foot deformity (pes cavovarus, pes planovalgus).

Presence of lower limb sensory impairment.

Presence of bladder and bowel disturbances.

\section{Data entry}

Data collected at each hospital site are deidentified and entered into the SDR-AUS Research registry's REDCap database. The database automatically distinguishes between retrospective and prospective patients according to the surgery date in relation to ethics approval date. For retrospective participants, data will be sorted from the medical records and entered accordingly. For prospective applicants, data will be collected and entered at baseline, at the time of surgery and immediate postoperative period and at 1, 2, 5 and 10 years post-SDR. Long-term $\mathrm{AE}$ data will be collected up to the date of termination from the study.

\section{Patient and public involvement}

Patients and public were not involved in the design of this protocol. There is public interest in this intervention; hence, the aim is to investigate the experience of SDR for Australian children and provide knowledge translation to patients and the public.

\section{Statistical analysis including sample size calculations}

It is anticipated that 10 children each year undergo SDR surgery in Australia with a larger proportion of children going overseas. The initial statistical analysis for gross motor outcome research will be undertaken when a sample size of 14 is achieved. This is based on a previous study where GMFM-66 scores increased from 69 (SD 8.9 ) to 76.2 (SD 8.5) in children classified as GMFCS I or II 1 year following SDR. ${ }^{26}$ This was determined using a paired t-test with a power of 0.8 and an alpha of 0.05 .

Descriptive data will be described using means and $\mathrm{SD}$, medians and ranges, or frequencies and proportions depending on the type and distribution of the data.

The primary research question (gross motor function) will focus on the results from the GMFM-66. Changes in gross motor function over time will first be assessed using the raw scores. When two time points are available, a paired t-test will be used. When three or more time points exist, then a mixed model approach will be used to account for the correlation between the time points. Given the likely small sample test or model, assumptions may be violated. Transformation of the data or nonparametric methods will then be utilised.

The GMFM-66 allows for comparison with reference curves over time allowing for the longitudinal assessment of the data. ${ }^{41}$ Each child's GMFM-66 score will be referenced against the average development pattern for children in his or her GMFCS level. After assigning centiles, a change in function between two percentile measurements will be determined using an $80 \%$ coverage probability. For children functioning at GMFCS I, a second percentile measurement has an $80 \%$ chance of being \pm 20 of the first measurement. For GMFCS level II, the cut-off is \pm 19.9 . At each time point, children will be classified as declined, increased or stable in their gross motor function based on these cut-offs. This technique has been described previously in the literature. ${ }^{41}{ }^{42}$ The frequency and proportion of children in each group will be examined over time and compared with expected outcomes from the CP population.

Surgeries performed overseas and in Australia will be analysed separately to minimise sampling bias. Differences in outcomes between procedure location (overseas vs Australia) will be compared using independent t-tests, Mann-Whitney $\mathrm{U}$ test or $\mathrm{X}^{2}$ test, depending on the type and distribution of the data.

The relationship between spasticity reduction and proportion of rootlets transected will be examined using scatter plots. Correlation (using Spearman's correlation coefficient) and simple linear regression will be used to further examine this relationship.

The remaining outcome measures (instrumented gait analysis, quality of life, Canadian Occupational Performance Measure and pain) will be analysed using paired t-tests when two time points exist. When three or more time points exist, then a mixed model approach will be used. Any missing data will be reported on as frequency and percentage. Given the likely small sample model, assumptions may be violated. Transformation of the data or nonparametric methods will then be employed.

All data will be analysed using Stata V.14.1 (StataCorp, College Station, TX, USA). Alpha will be set at 0.05 .

\section{DISCUSSION}

This protocol paper presents the background and study design for a national registry of multidimensional outcomes for Australian children undergoing SDR surgery. It will be the first Australian study to report on outcomes and AEs after SDR surgery. Limitations of this study are the risk of sampling bias and the lack of a control group. While the aim is for total population sampling, it is acknowledged that there is risk of sampling bias due to surgery location and potential missing data. Likewise, the value of a control group is recognised. However, there are challenges in identifying a suitable control group of adequate size for comparison due to the following: differences in children offered SDR versus those not offered SDR and very few children who are felt to be good 
candidates for SDR decline the surgery. The strengths of this study are in the selection of standardised outcome measures chosen which have been developed for the CP and/or paediatric population and capture the domains of the ICF, and the use of a purpose built database for documentation. Both allow for consistency in collection of data and potential for future collaboration with international sites.

\section{ETHICAL CONSIDERATIONS AND DISSEMINATION}

All participating Australian sites obtained individual ethics and governance approval from the Human Research Ethics committees and their respective Research Governance Units: HREC/16/SCHN/383 (NSW, SA, VIC, QLD); PRN: RGS0000000323 (WA).

All eligible participants and their families/carers are provided with a parent information sheet outlining the study and signed consent is obtained at the time of entering the study. Participant confidentiality and privacy will be strictly held in trust by all study personnel.

Results of this study will be published in relevant peer-reviewed journals and presented at relevant national and international workshops and conferences.

\section{Author affiliations}

${ }^{1}$ The Children's Hospital at Westmead, Kids Rehab, Sydney, New South Wales, Australia

${ }^{2}$ Child and Adolescent Health Service Western Australia, Perth, Western Australia, Australia

${ }^{3}$ Rehabilitation Department, Women's and Children's Hospital Adelaide Women's and Babies Division, North Adelaide, South Australia, Australia

${ }^{4}$ Department of Neurosurgery, The Children's Hospital at Westmead, Sydney, New South Wales, Australia

${ }^{5}$ Victorian Paediatric Rehabilitation Service, Royal Children's Hospital Melbourne, Parkville, Victoria, Australia

${ }^{6}$ Lady Cilento Children's Hospital, South Brisbane, Queensland, Australia

${ }^{7}$ Department of Paediatric Rehabilitation, Princess Margaret Hospital for Children, Perth, Australia

${ }^{8}$ University of Melbourne Melbourne Medical School, Melbourne, Victoria, Australia ${ }^{9}$ Hugh Williamson Gait Analysis Laboratory, Royal Children's Hospital Melbourne,

Parkville, Victoria, Australia

${ }^{10}$ Paediatric Rehabilitation Department, Women's and Children's Hospital Adelaide, North Adelaide, Australia

${ }^{11}$ Kids Rehab, Children's Hospital at Westmead, Westmead, New South Wales, Australia

${ }^{12}$ University of Sydney, Sydney Medical School, Sydney, New South Wales, Australia

Acknowledgements The authors acknowledge the National SDR Minimum Dataset Working Party for their involvement in the initial study design: JL, MaryClare Waugh, MW, Jill Rodda, FB, AS, Adrienne Forsang, Ray Russo, NB, Anna Gubbay, Dayna Pool, Kylie Aroyan, KM.

Contributors JL, NB, FB, AF, OL, KMcL, ER, AS, NS, PT, AT, MW and SP have contributed to the design of the protocol and to drafting and revising the manuscript, and have approved this version for submission. JL, FB, OL, NS, PT, MW and SP are responsible for recruitment and data management. In addition, NB has contributed to statistical methods and will be involved in interpretation of the results.

Funding Funding for this study was received from the Centre for Research Excellence in CP Victoria, as a one-off grant for start-up costs and initial development of the REDCap database used in this study, and a research grant was provided by The Cerebral Palsy Alliance (CPA) Research Foundation. Neither funding body has access to data or manuscripts.
Competing interests JL and NB have potential conflict of interest from partial funding provided by the CPA Research Foundation. All other authors have no conflict of interest. All authors are members of the research registry and have contributed equally to the project design and to the national meetings for this project since it commenced in 2015.

Patient consent for publication Not required.

Ethics approval All participating Australian sites obtained individual ethics and governance approval from the Human Research Ethics committees and their respective Research Governance Units: The Children's Hospital at Westmead NSW HREC/16/SCHN/383, SSA/17/SCHN/303; Princess Margaret Hospital for Children, WA PRN: RGS0000000323[JL3]; Lady Cilento Children's Hospital, QLD SSA/17/ QRCH/151; The Royal Children's Hospital, VIC SSA/17/RCH; and The Women's and Children's Hospital SA SSA/17/WCHN/174.

Provenance and peer review Not commissioned; externally peer reviewed.

Open access This is an open access article distributed in accordance with the Creative Commons Attribution Non Commercial (CC BY-NC 4.0) license, which permits others to distribute, remix, adapt, build upon this work non-commercially, and license their derivative works on different terms, provided the original work is properly cited, appropriate credit is given, any changes made indicated, and the use is non-commercial. See: http://creativecommons.org/licenses/by-nc/4.0/.

\section{REFERENCES}

1. Bax M, Goldstein M, Rosenbaum P, et al. Proposed definition and classification of cerebral palsy, April 2005. Dev Med Child Neurol 2005;47:571-6.

2. Mclntyre S. The continually changing epidemiology of cerebral palsy. Acta Paediatr 2018;107:374-5.

3. Oskoui M, Coutinho F, Dykeman J, et al. An update on the prevalence of cerebral palsy: a systematic review and meta-analysis. Dev Med Child Neurol 2013;55:509-19.

4. ACPR Group. Report of the Australian cerebral palsy register, birth years 1993-2009. 2016 https://www.cpregister.com/pubs/pdf/ACPRReport_Web_2016.pdf

5. Sanger TD, Delgado MR, Gaebler-Spira D, et al. Classification and definition of disorders causing hypertonia in childhood. Pediatrics 2003;111:e89-97.

6. Fairhurst C. Cerebral palsy: the whys and hows. Arch Dis Child Educ Pract Ed 2012;97:122-31.

7. Sanger T. Movement disorders in cerebral palsy. Journal of Pediatric Neurology 2015;13:198-207.

8. Aquilina K, Graham D, Wimalasundera N. Selective dorsal rhizotomy: an old treatment re-emerging. Arch Dis Child 2015;100:798-802.

9. Graham D, Aquilina K, Mankad K, et al. Selective dorsal rhizotomy: current state of practice and the role of imaging. Quant Imaging Med Surg 2018;8:209-18.

10. Peacock WJ, Arens LJ, Berman B. Cerebral palsy spasticity. Selective posterior rhizotomy. Pediatr Neurosci 1987;13:61-6.

11. Novak I, Mclntyre S, Morgan C, et al. A systematic review of interventions for children with cerebral palsy: state of the evidence. Dev Med Child Neurol 2013;55:885-910.

12. Grunt S, Fieggen AG, Vermeulen RJ, et al. Selection criteria for selective dorsal rhizotomy in children with spastic cerebral palsy: a systematic review of the literature. Dev Med Child Neurol 2014;56:302-12.

13. D'Aquino D, Moussa AA, Ammar A, et al. Selective dorsal rhizotomy for the treatment of severe spastic cerebral palsy: efficacy and therapeutic durability in GMFCS grade IV and V children. Acta Neurochir 2018;160:811-21.

14. Ingale $\mathrm{H}$, Ughratdar I, Muquit $\mathrm{S}$, et al. Selective dorsal rhizotomy as an alternative to intrathecal baclofen pump replacement in GMFCS grades 4 and 5 children. Childs Nerv Syst 2016;32:321-5.

15. Buizer Al, van Schie PEM, Bolster EAM, et al. Effect of selective dorsal rhizotomy on daily care and comfort in non-walking children and adolescents with severe spasticity. Eur J Paediatr Neurol 2017;21:350-7

16. Howick J, Chalmers I, Glasziou P, et al. OCEBM Levels of evidence working group. The Oxford 2011 levels of evidence 2011.

17. Gutknecht SM, Schwartz MH, Munger ME. Ambulatory children with cerebral palsy do not exhibit unhealthy weight gain following selective dorsal rhizotomy. Dev Med Child Neurol 2015;57:1070-5.

18. Langerak NG, Hillier SL, Verkoeijen PP, et al. Level of activity and participation in adults with spastic diplegia 17-26 years after selective dorsal rhizotomy. J Rehabil Med 2011;43:330-7. 
19. Langerak NG, Tam N, Vaughan CL, et al. Gait status $17-26$ years after selective dorsal rhizotomy. Gait Posture 2012;35:244-9.

20. Daunter AK, Kratz AL, Hurvitz EA. Long-term impact of childhood selective dorsal rhizotomy on pain, fatigue, and function: a casecontrol study. Dev Med Child Neurol 2017;59:1089-95.

21. Munger ME, Aldahondo N, Krach LE, et al. Long-term outcomes after selective dorsal rhizotomy: a retrospective matched cohort study. Dev Med Child Neurol 2017;59:1196-203.

22. Ailon T, Beauchamp R, Miller S, et al. Long-term outcome after selective dorsal rhizotomy in children with spastic cerebral palsy. Childs Nerv Syst 2015;31:415-23.

23. Engsberg JR, Ross SA, Collins DR, et al. Effect of selective dorsal rhizotomy in the treatment of children with cerebral palsy. $J$ Neurosurg 2006;105:8-15.

24. Josenby AL, Wagner $\mathrm{P}$, Jarnlo GB, et al. Functional performance in self-care and mobility after selective dorsal rhizotomy: a 10year practice-based follow-up study. Dev Med Child Neurol 2015;57:286-93.

25. MacWilliams BA, Johnson BA, Shuckra AL, et al. Functional decline in children undergoing selective dorsal rhizotomy after age 10. Dev Med Child Neurol 2011;53:717-23.

26. van Schie PE, Schothorst M, Dallmeijer AJ, et al. Short- and longterm effects of selective dorsal rhizotomy on gross motor function in ambulatory children with spastic diplegia. J Neurosurg Pediatr 2011;7:557-62.

27. Wright FV, Sheil EM, Drake JM, et al. Evaluation of selective dorsal rhizotomy for the reduction of spasticity in cerebral palsy: a randomized controlled tria. Dev Med Child Neurol 1998;40:239-47.

28. McLaughlin J, Bjornson K, Temkin N, et al. Selective dorsal rhizotomy: meta-analysis of three randomized controlled trials. Dev Med Child Neurol 2002;44:17-25.

29. Nordmark E, Josenby AL, Lagergren J, et al. Long-term outcomes five years after selective dorsal rhizotomy. BMC Pediatr 2008;8:54.

30. Steinbok P. Outcomes after selective dorsal rhizotomy for spastic cerebral palsy. Childs Nerv Syst 2001;17(1-2):1-18.
31. Tedroff K, Löwing K, Jacobson DN, et al. Does loss of spasticity matter? A 10-year follow-up after selective dorsal rhizotomy in cerebral palsy. Dev Med Child Neurol 2011;53:724-9.

32. Park TS, Liu JL, Edwards $C$, et al. Functional outcomes of childhood selective dorsal rhizotomy 20 to 28 years later. Cureus 2017;9:e1256.

33. Park TS, Edwards C, Liu JL, et al. Beneficial effects of childhood selective dorsal rhizotomy in adulthood. Cureus 2017;9:e1077.

34. Trost JP, Schwartz MH, Krach LE, et al. Comprehensive short-term outcome assessment of selective dorsal rhizotomy. Dev Med Child Neurol 2008;50:765-71.

35. Grunt S, Becher JG, Vermeulen RJ. Long-term outcome and adverse effects of selective dorsal rhizotomy in children with cerebral palsy: a systematic review. Dev Med Child Neurol 2011;53:490-8.

36. Langerak NG, Vaughan CL, Hoffman EB, et al. Incidence of spinal abnormalities in patients with spastic diplegia 17 to 26 years after selective dorsal rhizotomy. Childs Nerv Syst 2009;25:1593-603.

37. Golan JD, Hall JA, O'Gorman G, et al. Spinal deformities following selective dorsal rhizotomy. J Neurosurg 2007;106:441-9.

38. Spiegel DA, Loder RT, Alley KA, et al. Spinal deformity following selective dorsal rhizotomy. J Pediatr Orthop 2004;24:30-6.

39. Ravindra VM, Christensen MT, Onwuzulike K, et al. Risk factors for progressive neuromuscular scoliosis requiring posterior spinal fusion after selective dorsal rhizotomy. J Neurosurg Pediatr 2017;20:456-63.

40. Dindo D, Demartines N, Clavien PA. Classification of surgical complications: a new proposal with evaluation in a cohort of 6336 patients and results of a survey. Ann Surg 2004;240:205.

41. Hanna SE, Bartlett DJ, Rivard LM, et al. Reference curves for the gross motor function measure: percentiles for clinical description and tracking over time among children with cerebral palsy. Phys Ther 2008;88:596-607.

42. Bolster EA, van Schie PE, Becher JG, et al. Long-term effect of selective dorsal rhizotomy on gross motor function in ambulant children with spastic bilateral cerebral palsy, compared with reference centiles. Dev Med Child Neurol 2013;55:610-6. 